\title{
Enhanced Safety Message Transmission in High Density Traffic in Urban Region
}

\author{
Parminder Kaur $^{1}$, Deepinderjeet Kaur Dhaliwal ${ }^{2}$ \\ ${ }^{1}$ Research Scholar at DBU, Mandi Gobindgarh, $\mathrm{Pb}$. \\ ${ }^{2}$ Assistant Professor, DBU, Mandi Gobindgarh, $\mathrm{Pb}$.
}

\begin{abstract}
VANET is vehicular Ad-hoc network which is used for intelligent transport system for the drivers the ad-hoc network is used to transmit various types of message over the network. Safety message has to transmit for the security reasons on the vehicle and road transportation various routing protocols have been utilized for the purpose of message transmission. GPRS, AODV, DSR, PUMA these are various routing protocol utilizes for message transmission VANET scenario is used for mainly V2V and V2R purposes. The main issue in road density is high load because of this road message communication get overhead and due to less amount of network bandwidth to overcome this issue cognitive radio bandwidth can be utilize for data transmission by using channel sensing and message can be transmit through cognitive radio channels.
\end{abstract}

Keywords: VANET, AODV, DSR, PUMA, Security in VANET

\section{Introduction}

\subsection{VANET}

A VANET uses cars as mobile nodes in a MANET to create a mobile network. A VANET turns participating car into a wireless router or node which permitting cars 100 to 300 meters of one another to associate and make a system with a wide range. As cars drop out of the sign range and drop out of the system, different cars can join in, interfacing vehicles to each other so that a versatile system is made. It is evaluated that the main frameworks that will be this innovation are police and fire vehicles to speak with one another with the end goal of security. The availability is done among one vehicle to other vehicle and vehicle to street side foundation and vehicle or street side bases to the focal power in charge of the system support. The fundamental instrument for message exchange is the short range radios that are being introduced in any of the hubs.

\subsection{Types of Communication in VANET'S}

\subsubsection{Inter-vehicle communication}

The inter-vehicle communication configuration uses multihop multicast/broadcast to transmit movement related inarrangement over multiple hops to a gathering of beneficiaries. In intelligent transportation frameworks, vehicles require just worried with action out and about ahead and not be-rear. There are two sorts of message sending in between vehicle correspondences: naïve broadcasting and intelligent broadcasting .In naive broadcasting, vehicles send telecast messages intermittently and at regular intervals. Endless supply of the message, the vehicle overlooks the message on the off chance that it has originated from a vehicle behind it.

\subsubsection{Vehicle-To-Roadside Communication}

The vehicle-to-roadside correspondence design speaks to a single hop broadcast where the roadside unit sends a telecast message to every single prepared vehicle in the region. Vehicle-to-roadside correspondence setup gives a high data transmission join in the middle of vehicles and roadside units.

\subsubsection{Routing-Based Communication}

The routing-based communication configuration is a multihop unicast where a message is propagated in a multi-hop style until the vehicle conveying the wanted information is come to. At the point when the inquiry is gotten by a vehicle owning the coveted bit of data, the application at that vehicle immediately sends a unicast message containing the data to the vehicle it got the request from, which is then accused of the undertaking of sending it towards the question source.

\subsection{Challenges in VANET}

- A Speed-based Shortest Path Trip era has been included. Likewise, we can customize an edge between rapid road portions and separation to the destination, which might create a more drawn out however quicker way.

- Road Segments incorporates a rate confinement quality. For TIGER records, we create default values taking into account the State of California Current regulations, or let the client characterize them in an outside document.

- Decoupling the multi-path highlight from the path evolving highlight. At the point when numerous paths are accessible, every auto picks one path and keeps it (if accessible) for the whole trip.

- New Randomized Dijikstra shortest path algorithm. The first Dijkstra's calculation, given a begin and an end point, dependably chooses the same way, even in vicinity of numerous accessible ways with same weights.

\subsection{Characteristics of VANET}

- High Mobility: The nodes in VANETs usually are moving at high speed. This makes harder to predict a node's position and making protection of node privacy [2].

- Network topology: Due to high node mobility and random speed of vehicles, the position of node changes frequently. 


\section{International Journal of Science and Research (IJSR) \\ ISSN (Online): 2319-7064}

Index Copernicus Value (2013): 6.14 | Impact Factor (2015): 6.391

- Unbounded network size: VANET can be implemented for one city, several cities or for countries. This means that network size in VANET is geographically unbounded.

- Frequent exchange of information: The ad hoc nature of VANET motivates the nodes to gather information from the other vehicles and road side units. Hence the information exchange among node becomes frequent.

- Wireless Communication: VANET is designed for the wireless environment. Nodes are connected and exchange their information via wireless. Therefore some security measure must be considered in communication.

\subsection{Routing in VANET}

The main concepts of anchor-based routing in sensor networks have been adjusted to vehicular systems situations. GSR and SAR incorporate the street topologies in steering utilizing those ideas. In these conventions, a source processes the most limited street based way from its present position to the destination. Like RBVT, they incorporate the rundown of crossing points that define the way from source to destination in the header of every information parcel sent by the source. Be that as it may, don't consider the continuous vehicular movement, and thus, they could incorporate void streets or streets with system segments.

\subsection{Security Requirements for VANET}

- Authentication: Authentication is a major requirement in VANET as it ensures that the messages are sent by theactual nodes and hence attacks done by the greedy drivers or the other adversaries can be reduced to a greater extent.

- Message Integrity: This is very much requires as this ensures the message is not changes in transit that the messages the driver receives are not false.

- Message Non-Repudiation: In this security based system a sender cannot deny the fact having sent the message.

- Entity Authentication: It ensures that the sender who has generated the message is still inside the network and that the driver can be assured that the sender has send the message within a very short period.

- Message Confidentiality: It is a system which is required when certain nodes want to communicate in private.

\subsection{Applications of VANET}

According to the DSRC, there are over one hundred recommended applications of VANETs.

- Co-operative Collision Warning:Co-operative collision warning is an OBU-to-OBU safety application, that is, in case of any abrupt change in speed or driving direction, the vehicle is considered abnormal and broadcasts a warning message to warn all of the following vehicles of the probable danger.

- Lane Change Warning: Lane-change warning is an OBU-to-OBU safety application, that is, a vehicle driver can warn other vehicles of his intention to change the traveling lane and to book an empty room in the approaching lane. Again, this application depends on broadcasting.
- Intersection Collision Warning: Intersection collision warning is an OBU-to-RSU safety application. At intersections, a centralized node warns approaching vehicles of possible accidents and assists them determining the suitable approaching speed.

- Approaching Emergency vehicle:Approaching emergency vehicle is an OBU to-OBU public-safety application, that is, high-speed emergency vehicles (ambulance or police car) can warn other vehicles to clear their lane. Again, this application depends on broadcasting.

- Rollover Warning: Rollover warning is an OBU-to-RSU safety application. A RSU localized at critical curves can broadcast information about curve angle and road condition, so that, approaching vehicles can determine the maximum possible approaching speed before rollover.

\subsection{Cognitive Radio}

An intellectual radio is an intelligent radio that can be customized and arranged powerfully. Its handset is intended to utilize the best remote directs in its region. Such a radio naturally distinguishes accessible directs in remote range, then as needs be changes its transmission or gathering parameters to permit more simultaneous remote correspondences in a given spectrum band at one area.

\subsection{Spectrum Sensing}

The critical prerequisite of psychological radio system is to sense the spectrum hole. Subjective radio has a vital property that it distinguishes the unused range and shares it without unsafe obstruction to different clients. It figures out which parcel of the range is accessible and distinguishes the vicinity of authorized clients when a client works in authorized band.

\section{Approaches Used}

\section{GPSR PROTOCOL}

Greedy Perimeter Stateless Routing (GPSR) is one of the best examples of position based routing. GPSR uses closest neighbors information of destination in order to forward packet. This method is also known as greedy forwarding. In GPSR each node has knowledge of its current physical position and also the neighboring nodes. The knowledge about node positions provides better routing and also provides knowledge about the destination.On the other hand neighboring nodes also assists to make forwarding decisions more correctly without the interference of topology information. All information about nodes position gathered through GPS devices. GPSR overhead is defined as follows:

$$
O H_{G P S R}=\frac{p k_{\text {size }} \times E[n] \times E[h]}{E[t]}
$$

\section{Related Work}

Joanne Mun-Yee Lim et al [1] Vehicular communications are important to ensure emergency messages are transmitted on time to prevent accidents. Therefore, in recent years, various standardization bodies and automobile companies have developed vehicular ad hoc network (VANET) to 


\section{International Journal of Science and Research (IJSR) \\ ISSN (Online): 2319-7064 \\ Index Copernicus Value (2013): 6.14 | Impact Factor (2015): 6.391}

ensure public road safety. The current IEEE802.11p schemes utilize only traffic type to categorize priority levels. EPVS is evaluated in Vehicles in Network Simulation (Veins) with road traffic simulator, Simulation of Urban mobility (SUMO) using a realistic urban map. Simulations results show that the proposed EPVS results in lower average delay, in comparison with the default IEEE802.11p scheme.

Kalkundri Ravi et al [2]A Vehicular Ad Hoc Network (VANET) is a part of MANETs that is formed by wireless connections between cars. In VANETs, routing protocols and other routing related techniques must be adaptable to vehicular-specific capabilities and requirements. Along with the routing in VANET, message security is also one of the major concerns. Messages are critical and important like a warning message, so that the message must be authenticated which guarantee's the message integrity. The authentication of these messages is done with the help of an algorithm called Elliptic Curve Digital Signature Algorithm (ECDSA), which provides an efficient messageauthentication scheme.

Scott E. et al [3] A Vehicular Ad Hoc Network (VANET) is a part of MANETs that is formed by wireless connections between cars. In VANETs, routing protocols and other routing related techniques must be adaptable to vehicularspecific capabilities and requirements. Along with the routing in VANET, message security is also one of the major concerns. Messages are critical and important like a warning message, so that the message must be authenticated which guarantee's the message integrity. The authentication of these messages is done with the help of an algorithm called Elliptic Curve Digital Signature Algorithm (ECDSA), which provides an efficient message authentication scheme.

Hyun Yu et al [4]a routing protocol that works based on the real-time road vehicle density in order to provide fast and reliable communications so that it adapts to the dynamic vehicular city environment. In the proposed routing mechanism, each vehicle computes the vehicle density of the road to which it belongs by using beacon messages and the road information table. Based on the real-time road vehicle density information, each Vehicle establishes a reliable route for packet delivery.

Alwakeel, S et. al. [5] In VANET'S Safety messages is very much important so that it must have the highest assurance of delivery. But safety message can be rejected due to its low bandwidth. In this message we implement an approach to block minimum numbers of safety messages. But if you kept non safety message it can be penalized you. Through virtually partitioned VANET's bandwidth and by applying P-Persistent scheme to reduce message congestion an improved performance of message dissemination in VANETs can be achieved.

\section{Problem Formulation}

VANET is an extension of MANET; VANET is vehicular Ad-hoc network which is used for intelligent transport system for the drivers the ad-hoc network is used to transmit various types of message over the network. Safety message has to transmit for the security reasons on the vehicle and road transportation various routing protocols have been utilized for the purpose of message transmission. GPRS, AODV, DSR, PUMA these are various routing protocol utilizes for message transmission VANET scenario is used for mainly $\mathrm{V} 2 \mathrm{~V}$ and $\mathrm{V} 2 \mathrm{R}$ purposes. $\mathrm{V} 2 \mathrm{~V}$ is vehicle to vehicle communications and $\mathrm{V} 2 \mathrm{R}$ is vehicle to roadside communication. In various scenarios message transmission is done according to vehicle density on the road. On the basis of real time road density vehicles establish reliable route for the communication on packet delivery Ratio. The main issue in road density is high load because of this road message communication get overhead and due to less amount of network bandwidth to overcome this issue cognitive radio bandwidth can be utilize for data transmission by using channel sensing and message can be transmit through cognitive radio channels.

\section{Methodology}

VANET scenario is used for transmission of various messages is the vehicle communication. Various phases have been derived for proposed work that is described below in the form of the flow chart. Flow chart has the flow from the generation of the VANET to the analysis of the considered parameters through the various phases.

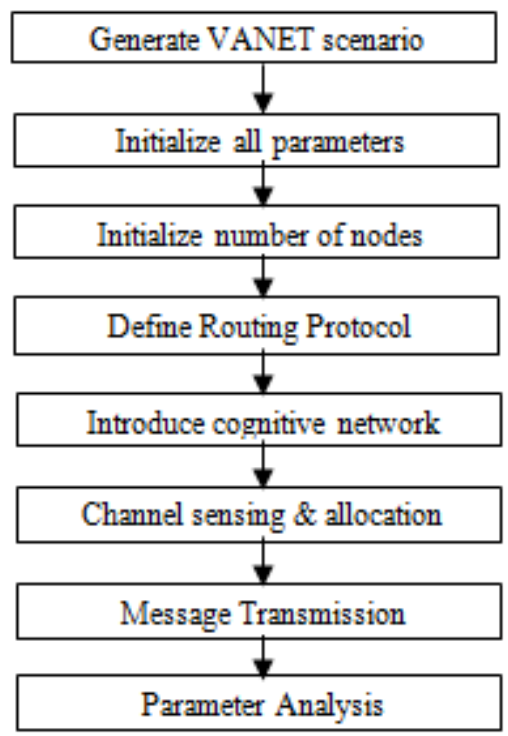

Figure 5.1: Flow of Purposed Work

Above given figure illustrate flow of purposed work that must be followed for achieving of desired objectives. This chart represents that transmission in VANET has been enhanced by using cognitive network in the purposed work

\section{Results and Discussions}

In the current study, various simulations have performed for achieving the results. NS2 simulator runs multiple times to achieve the results. In this research VANET scenario has initialized by defining traffic simulation no. of vehicle in the direction reverse to the direction of their mobility. 


\section{International Journal of Science and Research (IJSR) \\ ISSN (Online): 2319-7064}

Index Copernicus Value (2013): 6.14 | Impact Factor (2015): 6.391

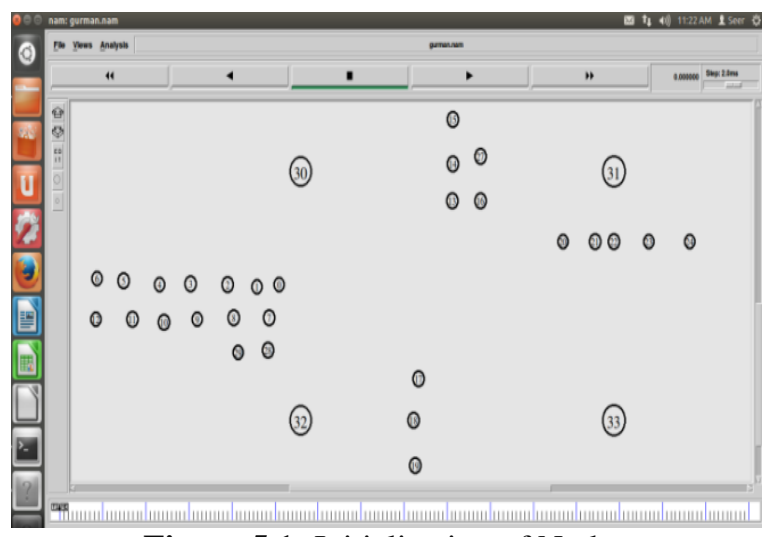

Figure 5.1: Initialization of Nodes

The figure 5.1 depicts behavior of nodes as vehicles. They are assumed to be in four different lanes and lane that is moving from left to right is assumed as a high density road. Node number 31, 32, 33, 34 acts as RSUs which communicate with vehicles.

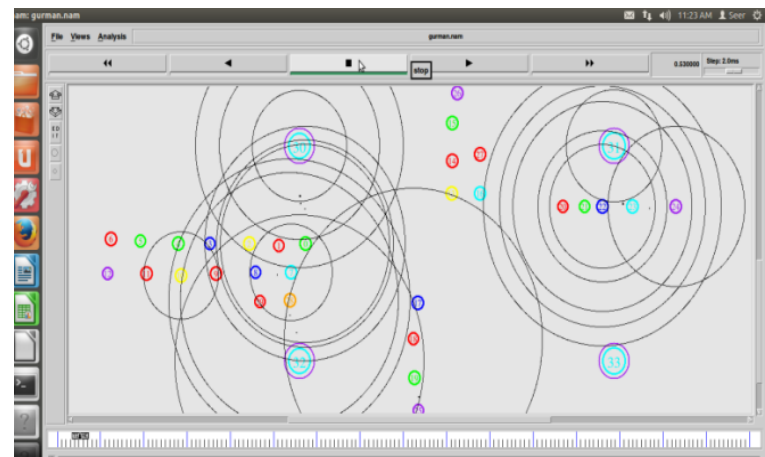

Figure 5.2: Communications between Nodes

Figure 5.4 shows that nodes of upper lane and lower lane crossed because of their green lights and nodes of left lane and right lane have stopped due to their red light.

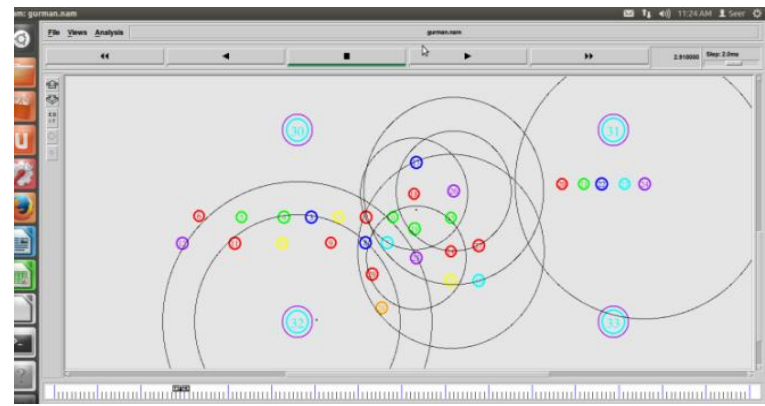

Figure 5.3: Movement of nodes

The figure 5.6 particularly shows that nodes of left lane which are now moving towards right now start communicate with their new RSU that is node number 31 and 33 .

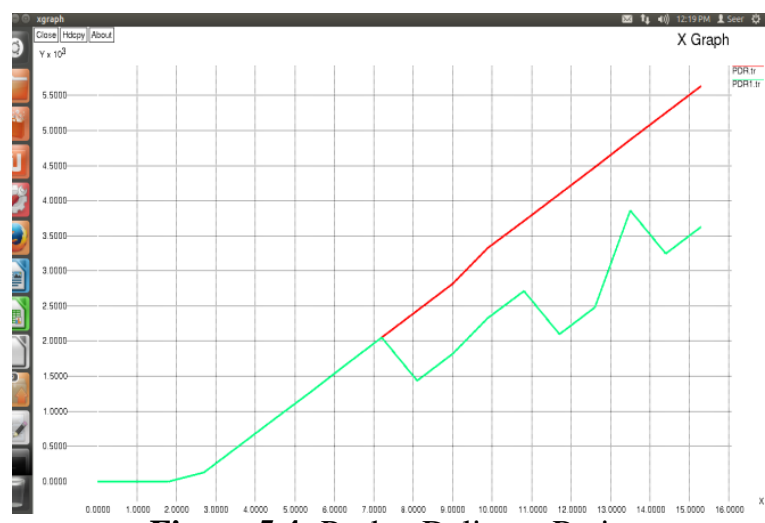

Figure 5.4: Packet Delivery Ratio

Figure 5.4represents the packet delivery ratio for network which shows that firstly when nodes just start communicate their PDR is idle then as they communicate this ratio goes on..

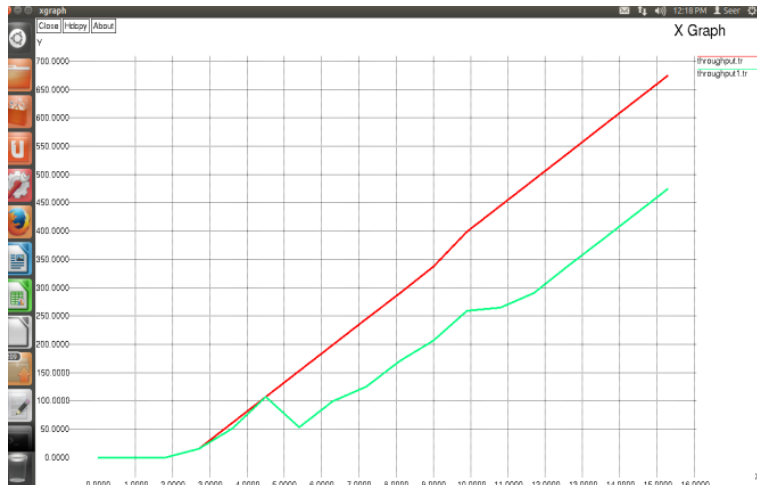

Figure 5.8: Throughput for purposed system

Figure 5.8 shows the calculated throughput for the nodes. Throughput defines packet delivery ratio per unit time.

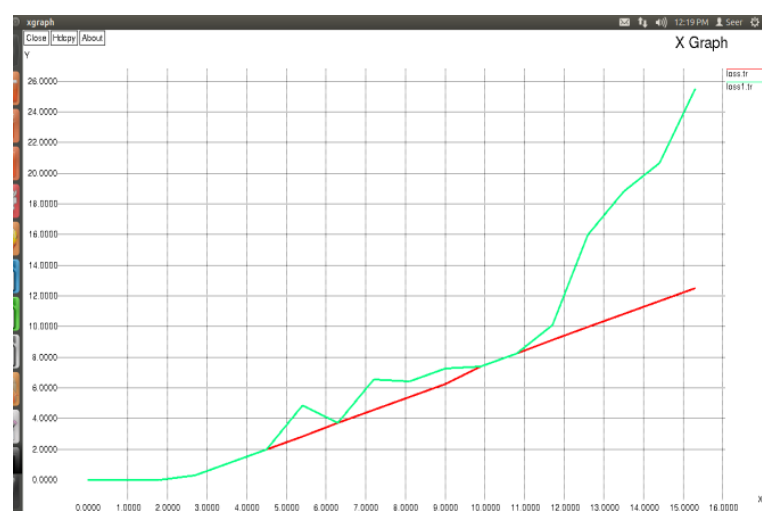

Figure 5.9: Packet Loss for purposed system

Packet loss is defined as the total no of packets loss from the system due to drop in the transmission. This is an essential parameter for performance evaluation of purposed system. 


\section{International Journal of Science and Research (IJSR) ISSN (Online): 2319-7064}

Index Copernicus Value (2013): 6.14 | Impact Factor (2015): 6.391

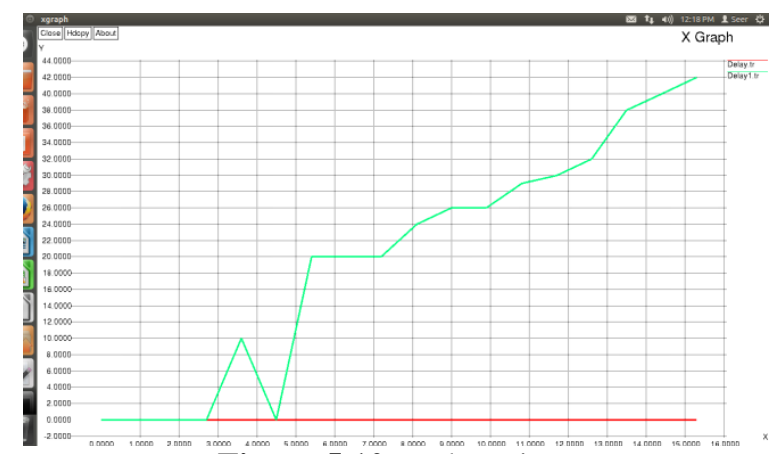

Figure 5.10: Delay Time

Delay is the time taken by bytes to reach its destination.

\section{Parameter Used}

QoS: Dedicated Short Range Communications (DSRC), specified under IEEE standard 802.11p. The IEEE 802.11 standard places the specifications for both the Physical layer (PHY) and for the Medium Access Control layer (MAC). The MAC extensions are mainly attention to get better security and QoS. The physical layer extensions mostly redefine the way in which the physical layer works. PHY and MAC layers of the VANET planned communication, Wirelesses Access in Vehicular Environment (WAVE), defined in IEEE 1609.x family of standards. The transmission technology for Intelligent Transportation System (ITS) can be typically classified into two categories, i.e. Vehicle-to Infrastructure communications (V2I) and Vehicle-to Vehicle communications (V2V).

A. Data Latency: Data Latency means time duration between issuing a message from sender until it is received by receiver vehicles. An important parameter to be considered in sending and receiving a data packet is transmission time delay, through which the throughput rate can be calculated. In order to calculate transmission time delay, the following steps are utilized.

\section{Del $=$ TTL $*$ distToInt $/ \operatorname{distToAp}$}

B. Efficient Bandwidth Utilization: The utilization of bandwidth estimation has a tremendous impact on system performance. If the bandwidth estimation is lower than that of network capacity, then the available bandwidth is underestimated and if the estimation bandwidth is higher than that of network capacity, then the available bandwidth is moreestimated.

$$
b_{\text {local }}=b_{\text {channel }} * t_{\text {idle }} / t_{\text {channel }}
$$

\section{Conclusion}

A VANET uses cars as mobile nodes in a MANET to create a mobile network. A VANET turns turn participating car into a wireless router or node which allowing cars 100 to 300 meters of each other to connect and create a network with a wide range. A cognitive radio is an intelligent radio that can be programmed and configured dynamically. Its transceiver is designed to use the best wireless channels in its vicinity. Due to traffic on high density roads, there is a high probability of loss on network so in this work Cognitive network is used which uses the channels and utilizes the free channel for transmission. In cognitive network channel sensing is used in which they sense for free channel and use that channel which lead to decrease the delay and increase the packet delivery ratio. This research is concluded on the bases of some quality of service parameters like PDR, Delay, and Throughput etc.

\section{Future Scope}

- This research can also be tested on large scale (i.e. for $n$ number of nodes. Where $\mathrm{n}$ can be any number).

- This work can also be tested with other protocols for comparative study.

- Same approach will be used to evaluate other parameters.

- In future this work can be done for huge network and on that scenario where there is traffic on all the roads at all the time and information is critical.

\section{References}

[1] Joanne Mun-Yee Lim "Cognitive VANET with Enhanced Priority Scheme" International Conf. on Telecommunications and Multimedia (TEMU), 2014, pp 116-121

[2] Kalkundri Ravi, "AODV Routing in VANET for Message Authentication Using ECDSA" IEEE Conf. on Communications and Signal Processing (ICCSP), 2014, pp 1389-1393

[3] Scott E, "AODV routing in VANET for message authentication using ECDSA" 2014, pp 1389-1393.

[4] Hyun Yu, “A VANET Routing based on the Real-time Road Vehicle Density in the City Environment"IEEE Conf. on Ubiquitous and Future Networks (ICUFN), 2013, pp. 333-337

[5] Alwakeel,S, “A virtual P-Persistent bandwidth partitioning manager for VANET's broadcast channel", International conf. on Multimedia Computing and Systems (ICMCS), 2014, PP 1212-1215.

[6] Varshney, "Security protocol for VANET by using digital certification to provide security with low bandwidth", International Conf. on Communications and Signal Processing (ICCSP), 2014, PP 768-772.

[7] Ghosh, T., "Congestion control by dynamic sharing of bandwidth among vehicles in VANET", International Conf. on Intelligent Systems Design and Applications (ISDA), 2014, PP 291-296.

[8] Gandhi, U.D, "Request Response Detection Algorithm for detecting DoS attack in VANET", International Conf. on Optimization, Reliability, and Information Technology (ICROIT), 2014, PP 192-194.

[9] Meriam, E.,"VANET adaptive and Reliable Broadcast protocol", International Conf. on Wireless Communications and Mobile Computing Conference (IWCMC), 2014, PP 237-243.

[10] Ali J. Ghandour a, Kassem Fawaz a, Hassan Artail a, Marco Di Felice b, Luciano Bononi, "Improving vehicular safety message delivery through the implementation of a cognitive vehicular network" IEEE Conf. on Telecommunications (ICT), 2013, PP 123-765.

[11] Srikanth Pagadarai, "Characterization of Vacant UHF TV Channels for Vehicular Dynamic Spectrum Access", IEEE Conf. on Vehicular Networking Conference (VNC), 2009, PP 1-8.

[12] Marco Di Felice, "Cooperative Spectrum Management in Cognitive", IEEE Conf. on Vehicular Networking Conference (VNC), 2011, PP 47-54.

\section{Volume 5 Issue 6, June 2016 www.ijsr.net}

